\title{
Design of Computer Experiments: a powerful tool for the numerical design of BAW filters
}

\author{
A. Reinhardt, F. de Crécy, M. Aïd \\ CEA, Leti, Minatec \\ Grenoble, France \\ alexandre.reinhardt@cea.fr
}

\author{
S. Giraud, S. Bila \\ XLIM-CNRS \\ Limoges, France
}

\author{
E. Iborra \\ Universidad Politécnica de Madrid \\ Madrid, Spain
}

\begin{abstract}
We apply Design of Computer Experiments methods to the simulation of piezoelectric stacks and the design of BAW resonators and filters. Through the example of DCS filters with two different technologies (Iridium and Molybdenum electrodes), we show that the definition and the exploitation of a metamodel can accurately replace acoustic simulations and allows therefore a much faster material stack design.
\end{abstract}

Keywords: Design of Computer Experiments, Resonator design, DCS filter.

\section{INTRODUCTION}

With the increasing complexity of filter and duplexer architectures, the acoustical design of BAW resonators needs to satisfy more and more constraints at the same time. This puts an increasing number of specifications on unitary resonators, like resonance frequency, but also effective coupling factor, quality factor, temperature sensitivity, etc. The fabrication process also puts many constraints. For this reason, defining a material stack for BAW resonators becomes a very complex task and designers need to find optimum material combinations to satisfy all these constraints at the same time. This can be achieved by the knowledge of the physical behavior of a resonator and sometimes by the use of an optimization algorithm. However, resonator specifications evolve very often during a filter design, as they are function of the overall architecture and of all the other elements included in the filter or duplexer, which can be passive elements as well as other resonators.

As an example, a possible design flow is first to design a filter function fulfilling specifications, then declining it in terms of filter topology and finally in terms of resonance and antiresonance frequencies for resonators. Starting from this first set of parameters, the designer needs to define a first material stack. In order to reach optimum quality factors and to reduce spurious resonances, dispersion curves of this stack need to be engineered, causing a modification of the initial material stack [1]. Usually, this optimized Bragg mirror causes a change of the effective piezoelectric coupling factor. Another

This work has been performed in the frame of the European Union funded MOBILIS project. possibility is that electrode thicknesses become so small that a high series resistance has to be expected, what is detrimental for insertion losses. Therefore, designers need to iterate between the electrical and the acoustical design until they come to a best compromise. These iterations require working many times on optimizing the piezoelectric stack.

In this work, we show that Design of Computer Experiments (DOE) methods can be applied to part of this design process in order to reduce the optimization time [2]. Resonator properties, like frequency and effective coupling coefficient can be modeled, within a given range of layer thicknesses, as simple polynomials. Thus, it becomes possible to replace full material stack evaluations by much simpler metamodels generated from only a few material stacks simulation with a minimum lack of precision. Once such metamodels have been generated and validated, optimization can be performed, even under multiple constraints. The advantage of such a metamodel is that a solution can be found rapidly if any constraints changes during the design process.

In a first part, we will present the methodology used to build metamodels and the limits of this approach. We will base ourselves on the design of a material stack suitable for the DCS filter band, which exhibits one of the most demanding set of specifications among RF telecommunication bands. In a second part, we will exploit the previously defined metamodels to the optimization of resonators that will be included in the filter structure, what will demonstrate the usefulness of this approach.

\section{DESIGN OF EXPERIMENTS}

\section{A. General considerations}

Our goal in this study is to define polynomial functions describing the dependence of resonance and antiresonance frequencies of simple BAW resonators with respect to layer thicknesses. For the sake of simplicity, in our example, only the most critical layers are considered: the two electrodes and the piezoelectric film. In order to design polynomial functions with a minimum number of simulations, we use DOE techniques: these will define one of the best sets of thickness combinations 
which need to be simulated in order to provide data to be fitted. These techniques also provide some tools to evaluate the quality of the fit and the accuracy of the function chosen. Eventually, an optimization process will be performed based on the functions generated.

Since we are building a DOE based on numerical experiments, some specific considerations need to be taken [4]. Especially, no random errors have to be considered. Therefore each simulation only needs to be performed once and experimental noise cannot affect the quality of the fit. The only source of uncertainty is the finite frequency sampling used in simulations and the imperfections inherent to the reference model.

\section{B. Model selection}

In a first step, we need to investigate the dependence of the resonance and antiresonance frequencies as a function of layer thicknesses in order to choose the best suited polynomial model. Figure 1. shows an example of dependence of the resonance frequency of a resonator based on a SiN/SiOC Bragg mirror, an AlN piezoelectric layer and two Mo electrodes versus the AIN thickness. This curve has been calculated using a simple Mason's model [5]. This figure also show results of linear, quadratic and cubic fits of this function calculated in the $1200-1700 \mathrm{~nm}$ range. As can be seen, at least a second order polynomial is needed to fit accurately this curve in the range of thicknesses of interest. The inset in Figure 1. shows magnified curves and helps estimating the fit error. This error can be directly evaluated from Figure 2. which compares three polynomials functions. Clearly, higher order functions provide better fits. However, if we reduce the design space, a lower order function can still provide enough precision. For the example of Figure 1., a second order polynomial function seems to provide enough precision in the $1200-1700 \mathrm{~nm}$ range for the AlN thickness. But this is only true for one set of electrode thicknesses. In order to ensure a precision sufficient enough over the whole design space, tests have shown that we need instead a third order polynomial function, even if second order functions would be more robust.

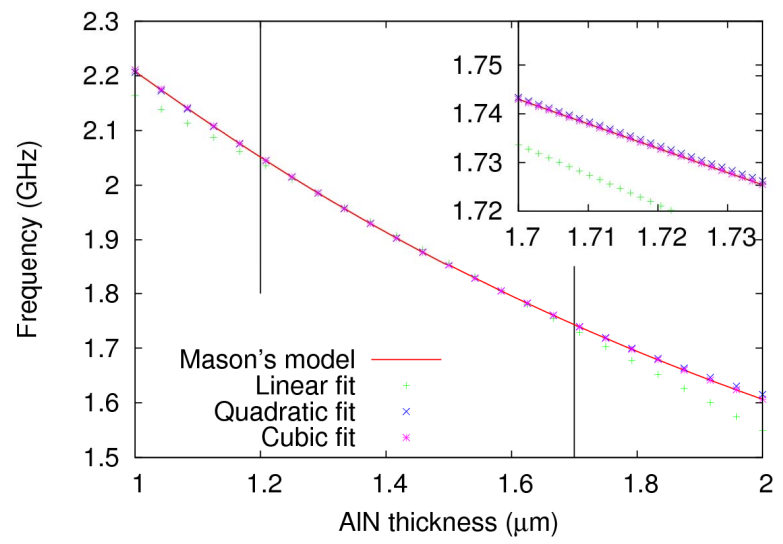

Figure 1. Dependence of resonance frequency on AlN thickness for a $\mathrm{Mo} / \mathrm{AlN} / \mathrm{Mo}$ piezoelectric stack and results of first and second order polynomial interpolations. Inset: magnification of curves close to $1.7 \mu \mathrm{m} \mathrm{AlN}$ thickness.

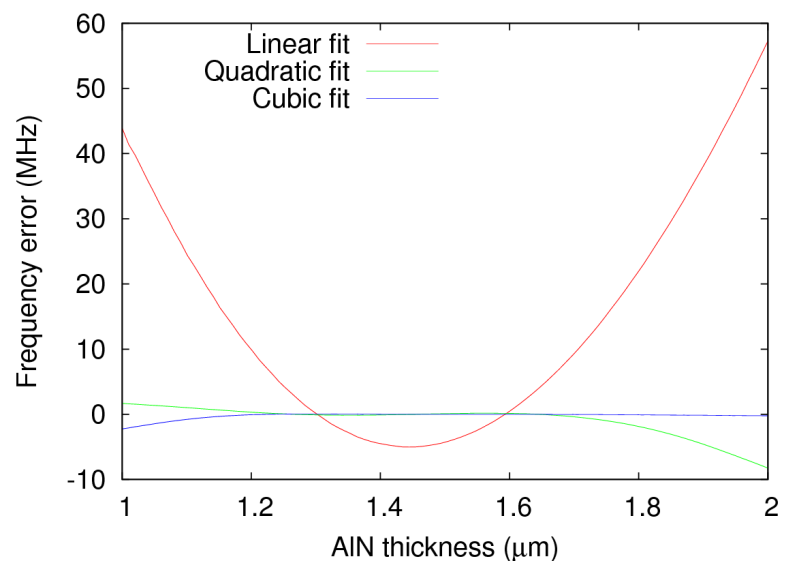

Figure 2. Estimation of the fit error for various polynomial functions.

\section{Design of numerical experiments}

Once the metamodel has been chosen, we need to design the numerical experiments on which the fit will be based. We assume initially a model of the most general form

$f=a_{0}+\sum_{i=1}^{3} a_{i} e_{i}+\sum_{i=1}^{3} \sum_{j=i}^{3} a_{i j} e_{i} e_{j}+\sum_{i=1}^{3} \sum_{j=i}^{3} \sum_{k=j}^{3} a_{i j k} e_{i} e_{j} e_{k}$

where the $a_{0}, a_{i}, a_{i j}$ and $a_{i j k}$ are the coefficients of the polynomial function, and $e_{1}, e_{2}$ and $e_{3}$ respectively stand for the thicknesses of the bottom electrode, piezoelectric layer and top electrode. Some of these coefficients may be removed if the analysis of variance (ANOVA) shows that they are statistically not meaningful. Therefore, we need to perform at least 20 simulations in order to determine the 20 coefficients. In practice, many more evaluations are performed, in order to improve and evaluate the quality of the fit as well as to estimate the residual error. For this study, we used 30 evaluations, but even more would be preferable.

As we are interested in building a metamodel which reproduces as closely as possible Mason's model simulations, we use a so-called D-Optimal design. Such a design generates a set of test points which minimizes the uncertainty on the polynomial coefficients obtained after fitting the simulated data with (1). Its major drawback is that we loose the orthogonality property of the design, i.e. it will be more difficult to evaluate the influence of one parameter when the others are kept constant.

\section{RESONATOR DESIGN}

\section{A. Filter design}

Following the previous considerations, we apply DOE methods to the design of resonators suitable for a DCS filter. Such a filter exhibits a very large relative bandwidth $(79 \mathrm{MHz}$ at a centre frequency of $1747.5 \mathrm{MHz}$ ) and needs therefore to be synthesized using BAW resonators with optimum effective piezoelectric coupling factors. Classical BAW resonators using 
Molybdenum electrodes are limited to $6.7 \%$ effective electromechanical coupling factors. More recently, work has focused on new electrodes material and Iridium has come as a good candidate for its high acoustic impedance and high conductivity. Therefore, it enables reaching effective coupling factors close to $7 \%$ [6]. In this work, we compare both materials.

As a first estimation, we want to design a material stack providing a resonance frequency corresponding to the centre frequency of the filter. We also want to define a stack close to it but with the antiresonance frequency corresponding to the filter centre frequency.

\section{B. Design of experiments}

Following some rough estimations of material stacks able to satisfy our specifications, we have defined some limits of the thicknesses we want to explore. These limits are also related to technological or design limitations (e.g. maximum sheet resistance allowed). These are summarized in TABLE I.

These sets of thicknesses are selected to provide frequencies that remain relatively close the targeted ones. This ensures that resonances remain within the frequency band in which the Bragg mirror is efficient. A second point is to ensure that we do not explore a domain which is too large, what could lead to a model exhibiting an increased error. However, the domain needs to be sufficiently large to contain all the possible solutions we may want to determine. So, at this stage, a compromise needs to be found between domain width and accuracy.

Figure 3. shows the set of 30 points provided by the Doptimal design for the Mo/AlN/Mo stack. These points are spread as uniformly as possible over the whole design space to provide a homogeneous prediction. An evaluation of the resonance and antiresonance frequencies for each set of thicknesses is performed by introducing the set of thicknesses in a Mason's model of the resonator. The same methodology is also applied to the Ir/AlN/Ir stack. These data are then fitted using (1). Before continuing, we need to test that the fit is valid. For this, we estimate the regression coefficient of our fits. In our example, we obtained a predicted $R^{2}$ (i.e. the regression coefficient which takes also into account the prediction power of the fit) of more than 0.99 , what shows an excellent agreement between the polynomial function and the values provided by the simulations as well as a high prediction power. However, this does not yet fully imply that the model is able to predict frequencies with the expected accuracy. We will have to check this property by evaluating the optimum points determined when exploiting the model and comparing their values to real Mason's model simulations.

TABLE I. DEFINITION OF THE DESIGN SPACES EXPLORED.

\begin{tabular}{|l|l|l|l|l|}
\cline { 2 - 5 } \multicolumn{1}{c|}{} & \multicolumn{2}{c|}{ Mo/AIN/Mo stack } & \multicolumn{2}{c|}{ Ir/AIN/Ir stack } \\
\cline { 2 - 5 } \multicolumn{1}{c|}{} & $\begin{array}{c}\text { Min } \\
\text { thickness } \\
(\text { nm })\end{array}$ & $\begin{array}{c}\text { Max } \\
\text { thickness } \\
(\text { nm })\end{array}$ & $\begin{array}{c}\text { Min } \\
\text { thickness } \\
(\text { nm })\end{array}$ & $\begin{array}{c}\text { Max } \\
\text { thickness } \\
(\text { nm })\end{array}$ \\
\hline Bottom electrode & 200 & 400 & 250 & 350 \\
\hline Piezoelectric layer & 1200 & 1700 & 1000 & 1500 \\
\hline Top electrode & 200 & 400 & 100 & 200 \\
\hline
\end{tabular}

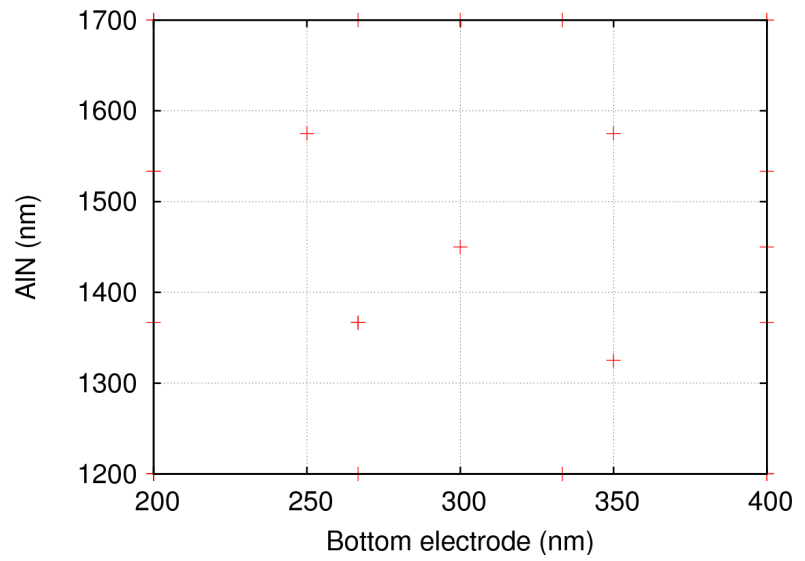

Figure 3. Position of test points along two of the design space coordinates for the $\mathrm{Mo} / \mathrm{AlN} / \mathrm{Mo}$ stack.

\section{Model exploitation}

After having built the metamodels, we exploit them. Our first example is the determination of the material stacks providing optimum effective piezoelectric coupling factors. Usually, one needs to perform many Mason's model evaluations to vary both top and bottom electrode thicknesses while adjusting the piezoelectric layer to keep the same resonance frequency and then generate an abacus showing the coupling factor as a function of the electrode thicknesses. From the known metamodels, we could solve the optimization problem analytically. However, since we are working with third order polynomials, solutions may not be obvious to find. Instead, we use a Simplex method which uses heavily evaluations of the metamodels. This iterative method builds a list of points converging towards a maximum of the desirability function defined as

$d\left(f_{R}, f_{A}\right)=\delta\left(f_{R}=f_{R, t \arg e t}\right) \cdot\left(\frac{f_{A}-f_{A, \text { min }}}{f_{A, \text { max }}-f_{A, \text { min }}}\right)$,

where $\delta\left(f_{R}=f_{R, \text { target }}\right)$ is a function which is equal to 1 when $f_{R}=f_{R, \text { target }}$ and decreases to 0 when $\left|f_{R}-f_{R, t \text { arg et }}\right|<250 \mathrm{kHz}$, $f_{A, \min }$ and $f_{A, \max }$ correspond to some arbitrary chosen limits for the antiresonance. This function is maximum when the resonance frequency reaches $f_{R, \text { target }}$, in our case $1747.5 \mathrm{MHz}$, while the antiresonance is at its highest value. Once this optimum found, we evaluate this point using Mason's model.

Results of the optimization, both in terms of thicknesses and of frequencies, are shown in TABLE II. As can be noticed, the agreement between the fitting polynomial and Mason's model is very good, with an average residual error below $1 \mathrm{MHz}$, what is within $+/-1 \mathrm{~nm}$ for any layer in the piezoelectric stack. This demonstrates the validity of our approach. In terms of time, these optima have been obtained in only a few minutes, instead of close to half an hour when optimizing the stacks by iterating simulations using Mason's model. 
In a second step, we want to calculate also loaded resonators. These resonators have an increased top electrode thickness to shift their antiresonance frequency down to the centre frequency of the DCS filter. For this, we fix the thicknesses of the bottom electrode and of the piezoelectric layer to their values given in TABLE II. Then, analytically, we calculate the thickness of the top electrode by using the polynomial expressions for the antiresonance frequencies of both $\mathrm{Mo} / \mathrm{AlN} / \mathrm{Mo}$ and Ir/AlN/Ir stacks. These results are also summarized in TABLE II. Again, the precision of the metamodel provides a good estimation of the Mason's model simulations, whereas this new optimum is obtained in only a few minutes. One can notice a slightly larger error for the $\mathrm{Mo} / \mathrm{AlN} / \mathrm{Mo}$ resonator, but by only reducing by $3 \mathrm{~nm}$ the top electrode thickness we can retrieve the targeted antiresonance frequency.

Finally, to show the versatility of the approach, we decide to design the Iridium resonators so that they match the same frequency specifications as the Molybdenum resonators. Since Iridium provides improved effective piezoelectric coupling factors, we know that a solution exists. We use the same optimization algorithm as previously, but this time the desirability function to optimize is

$d\left(f_{R}, f_{A}\right)=\left(1-\frac{\left|f-f_{R, t \text { arg } e t}\right|}{\delta f_{\max }}\right)\left(1-\frac{\left|f-f_{A, t \text { arg } e t}\right|}{\delta f_{\max }}\right)$,

in which $\delta f_{\max }$ is a normalization value defining the range of this function. The resulting thicknesses and frequencies are summarized in TABLE III. As for the previous example, the frequency shift between the configuration found using the metamodel and the validation using Mason's model remains within +/- $1 \mathrm{MHz}$.

TABLE II. RESULTS OF THE OPTIMISATION OF THE EFFECTIVE COUPLING FACTOR

\begin{tabular}{|l|l|l|l|l|}
\cline { 2 - 5 } \multicolumn{1}{c|}{} & \multicolumn{2}{c|}{ Mo/AIN/Mo stack } & \multicolumn{2}{c|}{ Ir/AIN/Ir stack } \\
\cline { 2 - 5 } \multicolumn{1}{c|}{} & $\begin{array}{l}\text { Unloaded } \\
\text { resonator }\end{array}$ & $\begin{array}{c}\text { Loaded } \\
\text { resonator }\end{array}$ & $\begin{array}{l}\text { Unloaded } \\
\text { resonator }\end{array}$ & $\begin{array}{c}\text { Loaded } \\
\text { resonator }\end{array}$ \\
\hline $\begin{array}{l}\text { Bottom electrode } \\
(\mathrm{nm})\end{array}$ & 274 & 274 & 266 & 266 \\
\hline $\begin{array}{l}\text { Piezoelectric layer } \\
(\mathrm{nm})\end{array}$ & 1690 & 1690 & 1290 & 1290 \\
\hline Top electrode (nm) & 231 & 272 & 114 & 132 \\
\hline $\begin{array}{l}\text { Resonance } \\
\text { frequency } \\
\text { (metamodel) (MHz) }\end{array}$ & 1747.5 & 1703.2 & 1747.5 & 1.6977 \\
\hline $\begin{array}{l}\text { Resonance } \\
\text { frequency (Mason) } \\
\text { (MHz) }\end{array}$ & 1748 & 1707 & 1749.5 & 1.698 \\
\hline $\begin{array}{l}\text { Antiresonance } \\
\text { frequency } \\
\text { (metamodel) (MHz) }\end{array}$ & 1793.7 & 1747.5 & 1798.7 & 1747.5 \\
\hline $\begin{array}{l}\text { Antiresonance } \\
\text { frequency (Mason) } \\
\text { (MHz) }\end{array}$ & 1794 & 1751.5 & 1800.5 & 1747.5 \\
\hline
\end{tabular}

TABLE III. IR/ALN/IR STACK MATCHING THE MO/ALN/MO STACK OF TABLE II.

\begin{tabular}{|l|l|l|}
\cline { 2 - 3 } \multicolumn{1}{c|}{} & \multicolumn{1}{c|}{$\begin{array}{c}\text { Unloaded } \\
\text { resonator }\end{array}$} & \multicolumn{1}{c|}{$\begin{array}{c}\text { Loaded } \\
\text { resonator }\end{array}$} \\
\hline Bottom electrode (nm) & 310 & 310 \\
\hline Piezoelectric layer (nm) & 1010 & 1010 \\
\hline Top electrode (nm) & 173 & 196 \\
\hline $\begin{array}{l}\text { Resonance frequency (metamodel) } \\
(\mathrm{MHz})\end{array}$ & 1747.5 & 1697.7 \\
\hline $\begin{array}{l}\text { Resonance frequency (Mason) } \\
(\mathrm{MHz})\end{array}$ & 1748.2 & 1696.7 \\
\hline $\begin{array}{l}\text { Antiresonance frequency } \\
(\text { metamodel) }(\mathrm{MHz})\end{array}$ & 1797.2 & 1745.7 \\
\hline $\begin{array}{l}\text { Antiresonance frequency (Mason) } \\
(\mathrm{MHz})\end{array}$ & 1798.2 & 1745 \\
\hline
\end{tabular}

\section{CONCLUSION}

We have applied design of computer experiments methods to the generation of metamodels able to represent the frequencies of a BAW resonator in the 1D approximation, for a given technology, as a function of layer thicknesses. Provided that the thickness range of interest is not too large, excellent agreement between the resonator simulation and its metamodel can be obtained. Examples involving a parallel design of resonators with Molybdenum and with Iridium electrodes have shown that the polynomial approximations can then be used to investigate resonator structures in the design space much faster than many $1 \mathrm{D}$ simulations.

Further work will consist in increasing both the precision and the extent of the design space, by using kriging or other techniques more suitable for design of computer experiments. Other computer experiments designs should also be tried in order to increase the accuracy and the design space extent of the models.

\section{ACKNOWLEDGMENT}

The authors want to thank Pr. P. Ozil, from the Institut Polytechnique de Grenoble, for his excellent tutorial on design of experiments who gave the idea for this work.

\section{REFERENCES}

[1] G.G. Fattinger, S. Marksteiner, J. Kaitila and R. Aigner, "Optimization of acoustic dispersion for high performance thin film BAW resonators", Proceedings of the 2005 IEEE Ultrasonics Symposium.

[2] T.J. Santner, B.J. Williams and W.I. Notz, "The design and analysis of computer experiments", Springer.

[3] T.W. Simpson, J.D. Peplinski, P.N. Koch and J.K. Allen, "On the use of statistics in design and the implications for deterministic computer experiments", Proceedings of DETC'97, Sept. 1997.

[4] J. Sacks, W. J. Welch, R. J. Mitchell, H. P. Wynn, "Design and analysis of computer experiments", Statistical Science, vol. 4, pp. 409-423, Nov. 1989.

[5] W.P. Mason, "A dynamic measurement of the elastic, electric and piezoelectric constants of Rochelle salt”, Phys. Rev. vol. 55, April 1939.

[6] M. Clement et al., "Aluminium nitride solidly mounted BAW resonators with iridium electrodes", Proceedings of the 2008 European Frequency and Time Forum, May 2008. 